\title{
Rehabilitative Soft Exoskeleton for Rodents
}

\author{
Juan Manuel Florez*, Manan Shah*, Eduardo Martin Moraud ${ }^{\dagger}$, Sophie Wurth ${ }^{\dagger}$, Laetitia Baud ${ }^{\ddagger}$, \\ Joachim Von Zitzewitz ${ }^{\ddagger}$, Rubia van den Brand ${ }^{\ddagger}$, Silvestro Micera ${ }^{\dagger \star}$, Grégoire Courtine ${ }^{\ddagger}$ and Jamie Paik ${ }^{*}$
}

\begin{abstract}
Robotic exoskeletons provide programmable, consistent and controllable active therapeutic assistance to patients with neurological disorders. Here we introduce a prototype and preliminary experimental evaluation of a rehabilitative gait exoskeleton that enables compliant yet effective manipulation of the fragile limbs of rats. To assist the displacements of the lower limbs without impeding natural gait movements, we designed and fabricated soft pneumatic actuators (SPAs). The exoskeleton integrates two customizable SPAs that are attached to a limb. This configuration enables a $1 \mathrm{~N}$ force load, a range of motion exceeding $80 \mathrm{~mm}$ in the major axis, and speed of actuation reaching 2 gait cycles/s. Preliminary experiments in rats with spinal cord injury validated the basic features of the exoskeleton. We propose strategies to improve the performance of the robot and discuss the potential of SPAs for the design of other wearable interfaces.
\end{abstract}

Index Terms - Lower limb exoskeleton, soft robotics, soft pneumatic actuators, rehabilitation robotics, wearable robotics, soft robotic interface.

\section{INTRODUCTION}

Severe neurological disorders can cause loss of sensation and activation of the lower extremities. To improve motor functions and restore locomotion, diverse treatment options are available. Among others, neurorehabilitation involving mechanical assistance with well-timed sensory inputs are being investigated extensively via animal test models [1], [2], [3]. For rodent models; widely used as test subjects in Spinal Cord Injury (SCI) research, throughout the training period, human trainers apply forces on the hip and knee joints to direct proper gait cycles. However instead of supplying symmetric, consistent, and constant forces, which is almost impossible, they exert mechanical stimulation to initiate movements to steer and achieve the ideal gait. While these methods have been highly effective [1], [2], [3], the patterns of force applied during manual assistance of the limbs are unknown, which necessitates proficient therapists who can optimally adapt training to the requirements of each subject based on their individual expertise and performing this task over thousands of times during a single training session.

Over the past decade, progress in developing robotic exoskeletons for neurorehabilitation has been largely aimed at providing repeatable, controllable and precise mechanical

\footnotetext{
* Reconfigurable Robotics Lab., Ecole Polytechnique Fédérale de Lausanne (EPFL), Lausanne, Switzerland

$\dagger$ Bertarelli Foundation Chair in Translational Neuroengineering, Center for Neuroprosthetics and Institute of Bioengineering, School of Engineering, Ecole Polytechnique Fédérale de Lausanne (EPFL), Lausanne, Switzerland

$\ddagger$ Courtine Lab., Ecole Polytechnique Fédérale de Lausanne (EPFL), Lausanne, Switzerland

* Translational Neural Engineering Area, The BioRobotics Institute, Scuola Superiore Sant'Anna, Pisa, Italy

Corresponding author: jamie.paik@epfl.ch

Abstract received October 31, 2014; Manuscript due February 15, 2015.
}

stimulation of human patients [4], [5], [6], [7] and adapted to patients [8], [9], [10]. However, for use in animal training this introduces challenges greater than just scaling down the force and size of the existing hardware and the training methods still remain intensively manual and laborious. Major issues are mechanism compliance and the attachment interface with the body of the subject; the anatomy of rodents is not only impractical for defining joint or pivot points and aligning the mechanism, but also rodents are not natural bipeds. Similarly it is not viable to conduct direct communication with rodents to specify and monitor the testing conditions. Therefore, functional rodent exoskeletons require hardware that acknowledges their particular anatomy as well as the extrinsic constraints and disturbances generated by voluntary or involuntary movements of the limbs. To our knowledge, there are two systems that address these challenges using motorized robotic arms. One of these, the Rat Stepper, is based on an elliptical machine where two PHANToM 1.0 haptic interfaces support each animal's paw [11]. In its later version, a treadmill was incorporated into the system while the haptic interfaces were attached to the animal's hindlimb ankles [12]. The latest design now replaces the two haptic interfaces with robotic arms and an active bodyweight support system. The Rat Car [13] allows quadruped gait on the floor without a treadmill. However, this system forces the animal to step in the sagittal plane. Both systems deliver the intended gait patterns but neither can replicate the degree of adaptation of human therapists as the systems are based on the paradigm that in order to provide stimulation, the rehabilitation system has to restrict functionality to predictable movements. Hence, mechanical constraints are imposed by the systems, limiting the interaction forces and velocities applicable to avoid leaving adverse re-education traces.

Recent developments in soft actuators [13], [14], [15] that are fabricated with polymeric elastomers exhibiting intrinsic compliance and elasticity have demonstrated the use of the material's softness to adapt efficiently to environmental constraints during manipulation [16], [17] or in bio-inspired robotics [18], [19]. The diversity of applications and forms that soft actuators can afford have nourished research where particular attention is on safety and adaptability for use in contact with fragile environments. Soft actuators can be tailored to virtually any specific embodiment [20] and have shown their potential in terms of forces and mechanical compliance requirements for applications in animals, [21] otherwise difficult to achieve with conventional actuation systems. Moreover, these flexible, elastic actuators are also being utilized by a growing number of research groups for assistive devices at the human scale [22], [23], [24], [25], [26], [27]. Our design of a soft pneumatic actuator (SPA)-based exoskeleton for rodents aims to allow interaction with fragile limbs and afford irregular 
gait patterns using the inherent compliance derived from the special properties of the soft material used. The main idea is to design hardware that exploits this novel actuation method for its capacity to adapt to extrinsic constraints [17], while satisfying the functional requirements of a wearable device for body weight-supported (BWS) treadmill training in rodent's who have undergone spinal cord injury. This paper presents an SPA-based exoskeleton for rodents and preliminary experimental results of this prototype in rodent locomotor training. The innovative exoskeleton is unique in design, using the customized SPAs described in Section II. We test its kinematic and dynamic capabilities in vivo, validating the performance of the device (Section III). We also reveal novel control algorithms to improve the outcome of training and subsequent rehabilitation in Section IV. The major contributions of this study are:

- The first reported prototype of an exoskeleton using SPAs with the design focus on safe physical interaction.

- A gait period adaptation control that integrates animal behavior information with whole body kinematics to adapt the mechanical stimulation on the rodent's hindlimbs. This control method can be applied to optimization of any irregular gait pattern regardless of the hardware capacity.

- The first measurement and quantification of the interaction force in gait training in rodents.

- A preliminary experimental validation of the efficacy of functional training for simple tasks.

\section{A Soft Pneumatic Actuator-Based Exoskeleton}

In neurorehabilitation research, exoskeletons are introduced to quantify and control the kinetic inputs dispensed to the patient. Results meant that therapies and progress could be better categorized and recorded to eventually provide time appropriate, controllable and safe sensory inputs to trigger the reshaping of spinal cord pathways. In all cases, the required force patterns to be applied to the subject are unpredictable and exoskeletons provide a solution for restraining functionality to predictable movements by imposing periodic tasks, hence increasing consistency.

Manual manipulation for locomotor training of rodents on a treadmill is difficult to achieve partly because of the scale and fragility of the limbs, but also because of unforeseeable motion activated by residual control of the limbs. Existing human exoskeletons are difficult to scale down to fit on $\sim 80 \mathrm{~mm}$ rat limbs. For this type of fragile application, the kinematic incompatibility creates unwanted forces that are more apparent at a lower interaction forces of $\sim 0.5 \mathrm{~N}$. For rodents, SPAbased systems provide a reliable and safe solution to achieve compliance at an interaction level that limits potential consequences of ill-modelled kinematic incompatibility.

\section{A. SPA Design Requirements}

The SPAs were designed to meet the desired characteristics for a rodent soft exoskeleton based on preliminary data collected in vivo. A paralyzed rodent with severe but incomplete SCI underwent functional locomotor training using a servocontrolled Body Weight Support (BWS) system and a motorized treadmill belt with manually assisted training sessions of
TABLE I

SOFT PNEUMATIC ACTUATOR AND EXOSKELETON PERFORMANCE.

\begin{tabular}{|c|c|}
\hline \multicolumn{2}{|c|}{ Exoskeleton's requirements } \\
\hline $\begin{array}{l}\text { Exoskeleton's Actuation } \\
\text { Speed [cycles/sec] }\end{array}$ & $>2$ \\
\hline $\begin{array}{l}\text { Exoskeleton's Blocked Force } \\
\text { at the end effector }[\mathrm{N}]\end{array}$ & 1 \\
\hline Force Modulation [KPa] & 0.01 \\
\hline Exoskeleton's & \\
\hline Range of Motion [mm] & $<80$ (in the major axis) \\
\hline \multicolumn{2}{|c|}{ Linear SPA specifications } \\
\hline $\begin{array}{l}\text { Air chamber size, } \\
\mathrm{W}[\mathrm{mm}] \times \mathrm{H}[\mathrm{mm}]\end{array}$ & $8 \times 8$ \\
\hline Air chamber length, Lc $[\mathrm{mm}]$ & 2 \\
\hline Number of chambers, $\mathrm{N}$ & 8 \\
\hline Wall thickness, $\mathrm{T}[\mathrm{mm}]$ & 3 \\
\hline Actuator height, Ho [mm] & 14 \\
\hline Actuator length, Lo $[\mathrm{mm}]$ & 65 \\
\hline Material & $\begin{array}{l}\text { Ecoflex } 囚 00-30 \\
\text { (Smooth-on, Inc.) }\end{array}$ \\
\hline Tube diameter, D [mm] & 2 \\
\hline Input pressure, $\mathrm{Pi}[\mathrm{kPa}]$ & $10-50$ \\
\hline Max blocked force $[\mathrm{N}]$ & 5.26 \\
\hline Max range of motion [mm] & $20(30 \%$ strain at $40 \mathrm{kPa})$ \\
\hline
\end{tabular}

20 min per session every other day for 4 weeks, starting 8 days post-SCI. Using a 6-axis force-torque sensor (ATI Nano17) attached to the therapist's thumb, forces were recorded to establish an interaction force profile between the therapist and the subject's limb. Equally, the kinematics of all of the rat's limb segments were monitored using a motion capture system (Vicon), as well as ground reaction forces (using a force plate) to establish an end-of-therapy goal trajectory. The trajectory obtained is depicted in Figure 7. The rat's limb trajectory at the end of training (i.e. 5 weeks after SCI) are approximated as ellipsoids, whereas in other studies [28], the reference trajectory was set by an experienced trainer moving the desired point of actuation manually. However, in order to allow the rodent to take full advantage of the compliance of the system, we guide the paw to a less constraining trajectory allowing the limb to adjust the profile. For the rest of the paper, we will only consider ellipsoidal trajectories for design evaluation.

It was established that the required trajectories imposed by the exoskeleton at the ankle point for physical training of a rodent's limb should be at least $80 \mathrm{~mm}$ in the forward direction and $50 \mathrm{~mm}$ in the vertical direction [29]. It was further determined that functional training did not require more than 2 cycles per second. A target ground reaction force of $1 \mathrm{~N}$ was also confirmed in this preliminary experiment. The overall design requirements for SPAs and the exoskeleton system are summarized in Table I.

In order to allow hip extension during the stance phase, necessary to initiate the swing phase, the initial exoskeleton design was attached tightly to the lower limb joints. However, several aspects of the animal's body complicated this. First of all, the animal's skin is very soft and composed of several layers that have no fixed anchoring or reference point relative to the harder muscles underneath. The skin could in fact slide over $10 \mathrm{~mm}$ from the initial point of measurement. Hence secure attachment onto the skin does not guarantee either positional or force transfer accuracy. Secondly, the animal's femur is a conical shape; the bone is thicker near the hip joint 
and thinner near the knee. Finally, we fixed the end effector to the ankle where the skin does not slip up to the knee joint, facilitating its attachment. The structure was designed as a whole limb exoskeleton that moves in parallel to the limb segments, and with adjustable femoral and tibial lengths to kinematically match the anatomy of the rat. This facilitates the addition of intermediate interaction ports in the future, like another SPA pushing at the level of the knee joint if attachment to this segment can be resolved and if considered helpful.

\section{B. Description of the Soft Exoskeleton}

The primary objective of this soft exoskeleton for rodents is to enhance training of physically paralyzed rodents to enable them to relearn the walking gait. This paradigm ultimately requires a force being applied on the leg to generate the hip-flexing motion with a desired output in terms of ground reaction force (GRF) of around $1 \mathrm{~N}$. Therapists encourage motion mainly in the sagittal plane, but in reality one should allow a certain abduction of the limb to prevent injuries or rehabilitation traces. The proposed exoskeleton design is depicted in Figure 1 and is composed of four main structures:

1) the Postural Structure that acts as a body weight support (BWS) system,

2) the Body Structure, that can be customized to fit onto different sized subjects of different anatomical dimensions,

3) the Active Part that is moved by the actuators including the attachments to the rodent's body,

4) the Actuators.

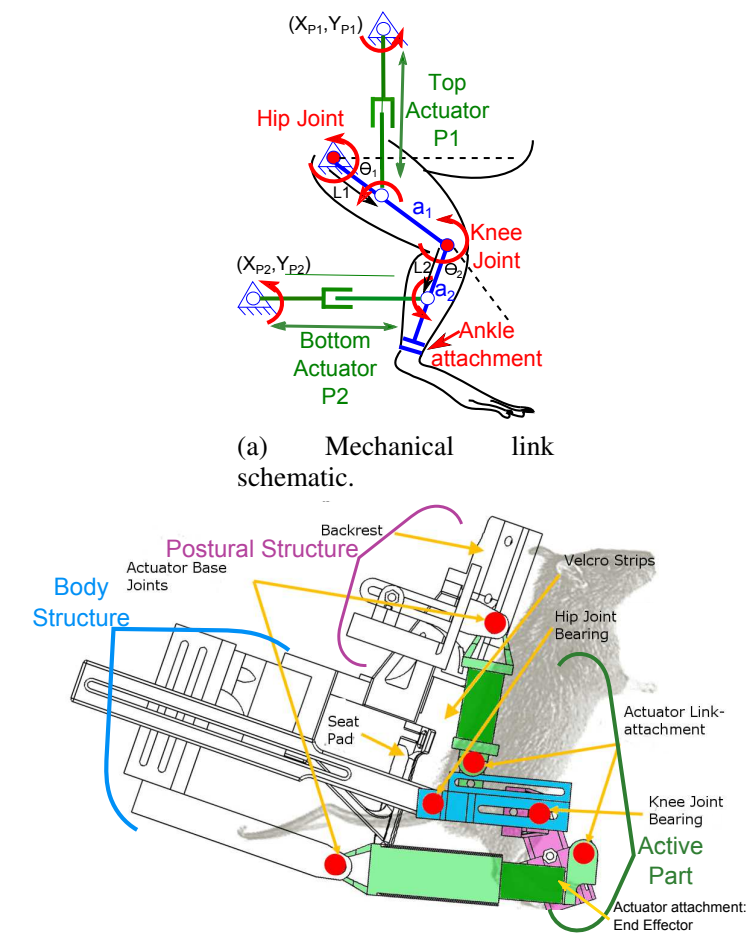

(b) Schematic of different parts of the SPA based exoskeleton.

Fig. 1. SPA-based exoskeleton for rodents. (a) Mechanical linkage schematic of the two-joint actuation mechanism representing the active part with the two linear SPAs attached to each segment at points L1 and L2 respectively and elongated to P1 and P2. (b) Schematic of the current prototype of the exoskeleton with adjustable links and passive joints (in red). Air tubing is not drawn.
1) Postural Structure: The postural structure is attached to a vertical BWS as described in [3]. It consists of a backrest attached to the upper body of the rodent using a passive Velcro jacket. This backrest is the frame of the orthetic device. The rodent is also attached to the exoskeleton via two Fabrifoam $16 \mathrm{~mm}$ BuddyStrap Velcro strips (Allenspach Medical); one around the mid-section of the torso and the other slightly above the hip-joint. The rodent's lower back rests on a vertical seat, which prevents the hip swinging backwards. The mounting method constrains the motion of the upper part of the body to an acceptable extent, ensuring a high level of control on lower limb movements, required for successful training of the rodent.

The overall mass of the complete exoskeleton is $600 \mathrm{~g}$, supported directly by the BWS. The active part (see later) weighs $150 \mathrm{~g}$ in total and the mass ratio of the moving hardware to the animal is about 0.5 .

2) Body Structure: Two passive 5-DoF arms extend from the two sides of the backrest and can be repositioned and locked to customize the orientation of the exoskeleton so that each subject can have the actuators just above the knee. The lower part of the body structure consists of two 4-DoF arms that position the bottom actuator behind the ankle joint. The position of each joint and the attachment points of each link, rigid or movable, can be adjusted to fine-tune the size and range of motion of the exoskeleton. Adjustability can be achieved while maintaining complete control over the motion of the Active Part to guarantee effective hip-flex motion.

A rigid pivot point that can be adjusted in all three $X, Y$ and $\mathrm{Z}$ directions is attached to the Postural Structure. This pivot point, at the hip joint bearing (see Figure 1), acts as a frame for the segment that runs parallel to the rodent's femur. This "femur-link" (Figure 1(b) coloured in light blue) holds a bearing in a separate mount that can be adjusted to match the position of the knee joint. The position of the bearing mount can also be adjusted along the length of the femurlink to adjust to the rodent's femur length. The same bearing mount holds the link corresponding to the tibia which is also of adjustable length, due to the presence of two bars that can slide relative to each other. The distal end of the "tibialink" (Figure 1(b) coloured in pink) is the end-effector of the exoskeleton and coupling at this level prevents overstimulation of the ankle nerves.

The tibia and femur links are matched to the rodent's limb segment lengths. The average tibia length (see a2 in Figure 1(a)) of an adult Sprague-Dawley rat is about $40 \mathrm{~mm}$ ([30]) and the average femur length (a1) is about $35 \mathrm{~mm}$. In this paper and in [29], Lewis rats are used, but we will assume the same body dimensions as Sprague-Dawley rats for this study. Hence, for construction of the prototype, the lengths of each actuator (L1 for the top actuator and L2 for the bottom one in Figure 1a) are bound by some limitations for practicality: $10 \leq L 1 \leq 30$ and $10 \leq L 2 \leq 35$. So the effective ranges of motion (in $\mathrm{mm}$ ) required for the actuators are between 14.3 and 42.9 for P1 and between 20 and 70 for $\mathrm{P} 2$.

3) Active Part: The exoskeleton achieves the required hipflex motion by moving the ankle along an ellipsoidal tra- 
jectory. This hip-flex motion requires controlled and welltimed periodic motion of the femur and tibia links. These links are independently actuated by two different linear SPAs for each leg. They are activated sequentially to a given amplitude with a particular phase difference generated by the controller described in the next section. The hinge joint links, positioned in the sagittal plane, hinder hip abduction/adduction movements. However, compliant attachments and soft linear actuators allow limb movement in the hip abduction and adduction directions by resisting the structural stiffness of the linear actuators to a certain extent. This in turn admits the adjustment of the trajectory of the ankle to some extent as they advance in their rehabilitation. This feature protects the animal in case of spastic movements.

A graphical user interface (GUI) was implemented in Labview with a first module for trajectory generation. This function allows, the geometric parameters of an ellipse for example, to be changed such as the lengths of the major and minor axis, a shift in $\mathrm{X}$ and $\mathrm{Y}$ directions of the center of the ellipse and a rotation in the $\mathrm{Z}$ plane around the center of the ellipse. The trajectory is then converted into actuator lengths $P 1$ and $P 2$ by computing the inverse kinematics of the exoskeleton (see below). An electro-pneumatic system, controlled directly by a virtual instrument (VI) in Labview, is connected to an integrated real time controller (National Instruments, cRIO-9082) with a $1.33 \mathrm{GHz}$ dual core processor and a LX150 FPGA. A pressure regulator (SMC, ITV101121F1N-Q) allows to digitally modify the pressure of each SPA and a solenoid valve is added to increase step response and as a security latch. This low level control system provides superior robotic support to accuracy and repeatability of inflation and deflation cycles of SPAs.

4) Actuators: In [21], we presented a first prototype of a soft exoskeleton for gait rehabilitation for spinalized rodents that exploits soft actuators. Soft actuators are designed to interact with unstructured environments [31], [32], [33]. They are highly compliant and can produce high power-to-weight ratios (over $10 \mathrm{~N}$ with a $100 \mathrm{~g}$ actuator) [34]. Polymer-based SPAs with embedded air chambers show innately low passive stiffness $(\sim 200 \mathrm{~N} / \mathrm{m})$ number, making them advantageous in tasks involving physical interaction with delicate body parts where the impedance of the machine, either passively or actively modulated, is required to be sufficiently low at times. Their high customizability and ease of fabrication [31], [32], [33], [34] is exploited thoroughly to optimize the geometries of the actuators to match the requirements for foot loading capacity, gait pattern and speed of stimulation. This can be achieved by varying the parameters shown in Figure 2a. The predominant SPA parameters optimized to generate the desired performance are the wall thickness $(T)$ and the chamber dimensions $(W \times H)$, which can affect the level of stationary strain with augmentation of input pressure. Note that the transient of the step response includes the dynamic effects of the pressure regulator and the solenoid valve that allows the air to inflate the chambers and hence the transitory response is not representative of the material properties nor the geometrical parameters of the chambers but will be used to predict the upper boundary of the actuator's bandwidth. Due to the similar requirements for the tibial and femoral actuators, a single design is used at both locations. The overall SPAs' geometric and dynamic characteristics are summarized in Table I. The fabrication time for each SPA is about 2 hours. This includes mold printing, curing materials and assembling the different parts. Installation takes less than 5 minutes, but to rebuild them, incorporating new design parameters requires about 3 hours to recalculate the necessary geometrical dimensions, create the molds and the actuators. Further details on the fabrication process can be found in [20] and in [34]. Once all the actuators are ready, it takes about an hour to assemble all the pieces.

The two linear SPAs are attached to both the Body Structure and links via simple hinge joints are illustrated in Figure $1 \mathrm{~b}$. The position of the hinge joint at the base of the actuators may be adjusted to align them as required. Also, their point of attachment along the length of the femur link may be adjusted to tune the length of the lever arm and hence the motion ratio. Figure 1a shows the direction of motion of the two actuators and links.

To safely interact with unstructured environments or fragile objects, an exoskeleton has to explicitly control its stiffness to avoid damage to the object or to the robot itself. But due to the bandwidth of the system and the wearer interaction this is not always achieved, even with the most sophisticated control strategy. Even though SPAs cannot match the bandwidth electrical motors can achieve [15], [27], [19], they have an embodied advantage of having a buffer zone, convenient for certain applications such as the lightweight soft exosuit [24], navigation robots [35], [18], [36] or grippers [16], [27], [37] because they compliantly adapt to the environment without having to specifically address trajectories. For this study we exploit this aspect of SPAs to address the challenging problem of assisting fragile animal limbs by controlling the pressure inside the SPA chambers and computing inverse dynamics to determine the input signal to be sent to each pressure regulator. This mode of control enables the characterization of stiffness depending on the load. By changing the attachment point of the proximal part of the SPA and adjusting the effective range of the pressures we can regulate the stiffness value. Stiffness is not actively adapted throughout the trajectory for experiments described in Subsections III-A-D, but the speed of actuation is controlled in the experiments in Subsection II-E, modifying the impedance of the device.

\section{Characterization of the Exoskeleton}

In a $2 \mathrm{D}$ input space with a two-joint mechanism actuated by two linear SPAs attached to the points described by the relative vectors $L 1$ and $L 2$ and given the desired co-ordinates, the inverse kinematics problem is reduced to finding the two lengths $P 1$ and $P 2$ involved. We define the first angle, $\theta_{1}$, as the angle between the first arm (femoral arm) of length $a 1$ and the hip joint (ground). The second angle, $\theta_{2}$, is the angle between the first arm and the tibia segment of length $a 2$. The schematic of the system is illustrated in Figure 1a. Since the proximal points of the actuators are adjustable, their position is defined as $\left(x_{P 1}, y_{P 1}\right)$ and $\left(x_{P 2}, y_{P 2}\right)$. The actuator lengths 


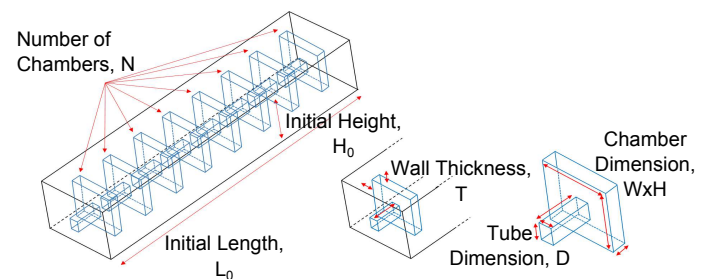

(a) Geometrical parameters for linear SPAs. The chamber dimension cross section area $W \times H$, and wall thickness, $T$ are the most influential parameters in displacement and blocked force performance.

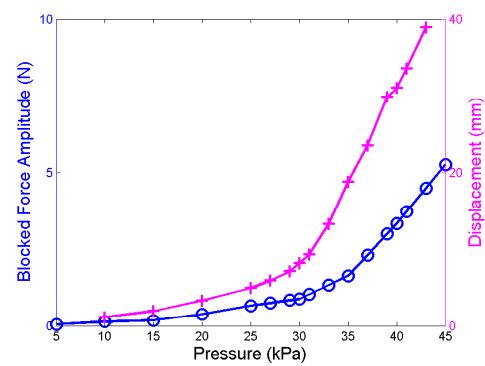

(b) Blocked force and linear displacement vs input pressure of the exoskeleton's linear SPA.

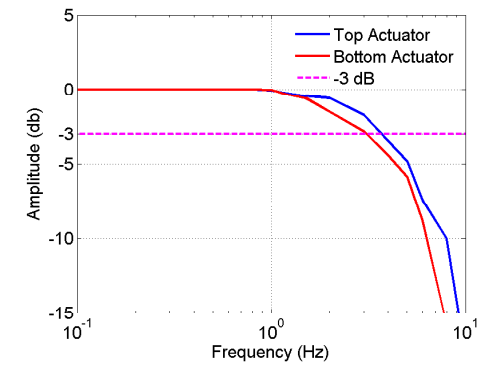

(c) Top and Bottom SPA magnitude response. The bandwidth (crossing point with the $-3 \mathrm{~dB}$ magenta line) is about $3 \mathrm{~Hz}$ and $3.5 \mathrm{~Hz}$ for each SPA respectively.

Fig. 2. Linear SPA parameter description and performance evaluation of single actuators in terms of displacement and blocked force vs pressure and bandwidth.

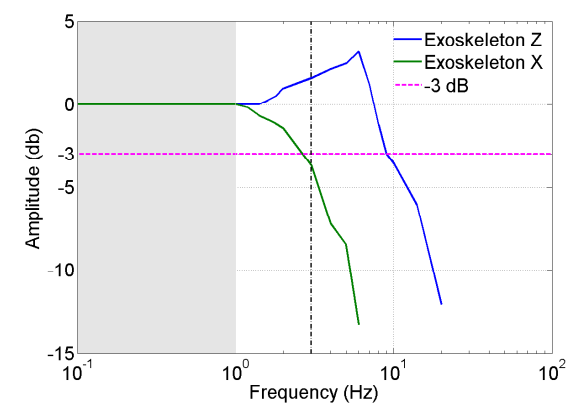

Fig. 3. Magnitude response of the end-effector displacement of the exoskeleton with no load. The bandwidth is about $3 \mathrm{~Hz}$ for the exoskeleton. Measurements were taken in the sagittal plane: $X$ (anterior-posterior in green) and $\mathrm{Z}$ (vertical in blue). The bandwidth of the human tracking performance of a continuously varying input signal is shaded in grey [38].

can be computed in terms of the projections of points $a 1$ and $a 2$ in the ground reference $\left(\tilde{x_{a 1}}, \tilde{y_{a} 1}\right)$ and $\left(\tilde{x_{a 2}}, \tilde{y_{a} 2}\right)$ :

$$
\begin{aligned}
& P 1=\sqrt{\left(\tilde{x_{a 1}}-x_{P 1}\right)^{2}+\left(\tilde{y_{a} 1}-y_{P 1}\right)^{2}} \\
& P 2=\sqrt{\left(\tilde{x}_{a 2}-x_{P 2}\right)^{2}+\left(\tilde{y_{a}}-y_{P 2}\right)^{2}}
\end{aligned}
$$

The workspace of the mechanism is computed and illustrated in Figure 7 and is confirmed to enclose the desired trajectories defined in Section II-C as requirements. SPAs add a little resonance $(3 \mathrm{~dB})$ in the vertical direction. Also, we used OpenCV tracking software to measure the trajectory of the end effector of the exoskeleton. We evaluated the bandwidth of the open loop system in the sagittal plane as shown in Figure 3. The bandwidth ( $-3 \mathrm{~dB}$ point) of the SPA-based system was about $3 \mathrm{~Hz}$. The hyper-elasticity of the exoskeleton is capable of producing at least 2 cycles / $\mathrm{s}$ of mechanical stimulation which is faster than the requirements for normal gait cycles as reported in Table I.

\section{Rodent-Exoskeleton interaction forces}

Interaction effects between live subjects and robotic devices can be measured indirectly through effort assessment such as Electromyography (EMG)[39], [40] or metabolism monitoring [41] or directly through transducers placed in the interface between the two systems [42], [43]. A preliminary test was performed to compare the force/torque levels of interaction between the exoskeleton and the ankle of the subject to the levels of force applied during manual assistance. A six axis force/torque sensor (ATI Nano17) was placed at the ankle attachment. The measurements were compared to recordings of forces measured through a device composed of a fixture attached to the thumb of the therapist that holds the same force/torque sensor at the level where they make contact with the rodent's leg. The rodent was stimulated with Electrical Epidural Stimulation (EES) as in the conventional BWS treadmill training method above.

This setup clearly increases the inertia of both the moving part of the exoskeleton and of the therapist's thumb modifying the "normal" way of performing the gesture. Nevertheless, it allows comparison of the interaction forces levels between the manual and exoskeleton enabled methods, as will be reported in Section III-D. The bandwidth of the exoskeleton is also reduced to $<1 \mathrm{~Hz}$ limiting its applicability for automation of training.

An alternative way of closing the loop for control of the mechanical stimulation is to measure the kinematics of the coupled system and adapt the trajectory of the robotic device depending on the error between the desired trajectory and the measured one. This would imply that the tracking error is proportional to the interaction force disturbance applied by the subject. This assumption is potentially dangerous for the subject attached to a rigid robotic device. For a SPA actuated device this problem is mitigated due to the material compliance.

\section{E. Gait period adaptation control scheme of the Exoskeleton}

We designed an active assistive device to supply mechanical stimulation to achieve a preprogrammed gait. The main motivation of such assistance is not to impose motion but to assist and motivate appropriate supraspinal and peripheral activation that helps induce CNS plasticity. On the other hand, continuously compensating for trajectory errors might decrease the involvement of the subject in the task [28], [44].

The material properties of the SPA allow the system to be inherently compliant and to guide the rodent's leg while acting like a damped spring if it deviates from the desired trajectory. Therefore, the control effort of the system is lower and the subject is able to override the task. This operating mode is inspired by manual assistance wherein the human therapist is able to actively guide the rodent's limb and to react when the rodent has residual control. 


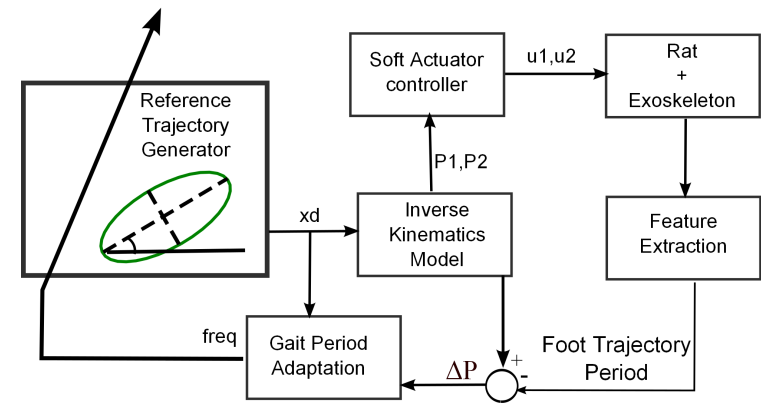

Fig. 4. Principle of the gait period adaptation controller using an ellipsoidal reference trajectory implemented in cartesian-space.

Another issue is that neither the velocity profile of the foot during a step nor its duration is constant. Despite this irregular gait of the subject, it is crucial to synchronize the stimulation from the exoskeleton with this gait cycle. Otherwise we have adverse effects on the therapy. For example, if the device is slower than the subject's intended movement, a disturbance in form of a virtual viscous field is added to the load. If the system is faster than the subject, it would co-contract its muscles impeding the motion.

To avoid over-constraining the rodent's limb, we minimized the control effort necessary for reducing the trajectory error all along the path. If the system to control is unpredictable and capable of reacting to every change in the control effort, the limitation of applied corrections can reduce the chance of over-constraining the limb. This can also avoid compensatory strategies during walking response such as excessive cocontraction or slacking.

One way of implementing such a compliant assistive controller is to measure the cyclic offset between the period of imposed trajectory and the actual period of the subject's reactive motion [45]. The closer the period motion of the coupled system is to the input period, the easier it is for the rodent to move, and the higher the force that is transmitted to the ground loading the foot. This technique can be applied to any co-manipulated device where there is an imposed motion and a voluntary motion to generate a synergistic transmission of forces. The rationale behind this technique is to adapt the period cycle after cycle, since the synergy function between the robot (or the human therapist) and the rodent is currently undetermined. Although, this approach does not turn the system transparent, it contributes to reduce discrepancies in the synchronization of the trajectory performed by the rodent and the one applied by the exoskeleton to $<10 \%$ of the period, which could create unwanted effects on the interaction port. It is also in accordance with the idea to limit the control effort to once per cycle but provide assistance all along the trajectory, encouraging the learning of stepping in the long term instead of fully assisting in continuous mode during the cycle.

A gait period adaptation controller such depicted in Figure 4 was developed and tested. Force and trajectory measured using the Vicon motion capture system and the 6-axis force plate allowed to extract features of the movement such as the gait's frequency. A controller implemented within a multithreaded C++ code (Visual Studio 2010, Microsoft) running on a quad core Microsoft Windows 7 computer allows detection of the gait's frequency and triggers control inputs to actuate the exoskeleton. These signals are generated through an RZ5 processing unit (Tucker-Davis Technologies) connected to an MS16 Stimulus Isolator (Tucker-Davis Technologies). The input is sent to the electro-pneumatic system described in Section II-B that computes the desired actuator lengths through an inverse kinematics algorithm and sends commands to the pressure regulators and solenoid valves adapting the period of the signal inflating the SPAs. In this experiment, no EES stimualtion was used.

\section{PRELIMINARY EXPERIMENTAL VALIDATION}

The design of the presented prototype is based on the training of rodents with neurological disorders and currently demonstrates sufficient range of motion (see Figure 7). The softness introduced by the actuation method makes it suitable for interaction with fragile environments. A Lewis rat (female, $200 \mathrm{~g}$ ) with complete loss of gait function due to severe contusion on the spinal cord was chosen. Contusion models were preferred because they can have predictable and consistent functional outcomes hat allow testing of potential therapies and are considered to resemble human SCI more closely than transection approaches. In terms of the end effector motion for various gait trajectories, the soft exoskeleton delivers a cyclic gait pattern $>80 \mathrm{~mm}$ in the major axis, and has the capacity to produce up to $1 \mathrm{~N}$ on each foot with mechanical stimulation. These figures are only a representative of the current prototype, which can be scaled to up $(\times 2$ without changing the hardware, and more with some component replacement) with simple SPA changes. The geometrical discrepancies between rodents and even within a single rodent before and after surgery, are significant. We have considered these subject biometrics (size, weight, gait pattern, receptiveness to certain therapies, health level post surgery) for the soft exoskeleton design. Consequently, three test rodents were successfully fitted to the soft exoskeleton without changing any hardware components to satisfy the defined force and range of motion criteria. However, the test results must be post-calibrated (the peaks match but the shape and response of the modulation need to be calibrated per subject) based on the attachment position of the exoskeleton, attachment of the BWS, the subject's health and motivation level. As the first step, in Subsection III-A, we tested the exoskeleton attached to a passive limb (without EES or pharmacological enablers) describing trajectories without contact with the ground to verify guidance of the ankle's trajectory. Next, as described in Subsection III-B, we verified the modulation of the forces and trajectories of the exoskeleton on the subject: we measured the ground reaction forces and verified the relation between changing the parameters of the ankle's planned trajectory and the force output. Subsequently, as described in Subsection III-C, we conducted experiments on a moving treadmill belt mimicking a conventional training setup and comparing it to static environment response. Finally, Subsection III-E describes the implementation of a frequency adaptation controller that allowed us to characterize the effect of period error in ground reaction force level. 
A. Active guidance of a passive limb without floor contact: Airwalk tests

The airwalk test is a preliminary experiment to show the capacity of the developed prototype to guide the rodent limb along a given trajectory within the operational space of the exoskeleton. For this experiment the rodent was attached to an automated, servo-controlled BWS system (Robomedica) without touching the treadmill. No EES or pharmacological agent was used during this experiment. The exoskeleton was attached to the ankle of the animal using a rigid cable tie lined with neoprene. Different ellipsoidal trajectories were imposed by the exoskeleton selecting the parameters of the form and orientation of the ellipse in the Labview GUI. The displacements of the ankle in three dimensional space were measured with the Vicon system. The same trajectory was applied without attaching the exoskeleton to the rodent and recorded. It was processed offline through OpenCV object tracking software and converted to units of length using a reference placed in the video.

In Figure 5, we illustrate three different representative cases of a combination of ellipse parameters each for four full consecutive cycles. The path followed by the ankle reveals that the soft exoskeleton succeeds in pushing the foot to follow the predefined trajectory. Contrary to a rigid body robot, the soft exoskeleton does not fully impose the trajectory but it allows deviation from the desired path hence improving gait cycle synchronization and comfort. Although softness in the actuation method implies a decrease in the precision of the following of the trajectory, since the rodent can backdrive the structure, it is also closer to the manual training procedure since the therapist does not guide the limb throughout the entire trajectory, but only as needed.

To identify which features are relevantly reflecting tuning of leg movements, we performed a study computing kinematic variables that provide a description of trajectory following of the ankle. For these examples, both the angle of the ellipse and the length of the major axis have an influence on the trajectory measured through the motion capture system. We show that increments of major axis length and/or changes in the angle of rotation of the major axis with respect to the horizontal plane (angle of ellipse) led to a graded and consistent modulation of cyclic patterns in all the cases within a range of $20-80 \mathrm{~mm}$.

Even though the exoskeleton closely follows the desired trajectory in open loop as shown in Figure 5, when connected to the rodent, the residual control of the subject acts as an output disturbance. Also, the rodent's leg involuntary movements are inevitable. The proposed structure, specifically the compliance

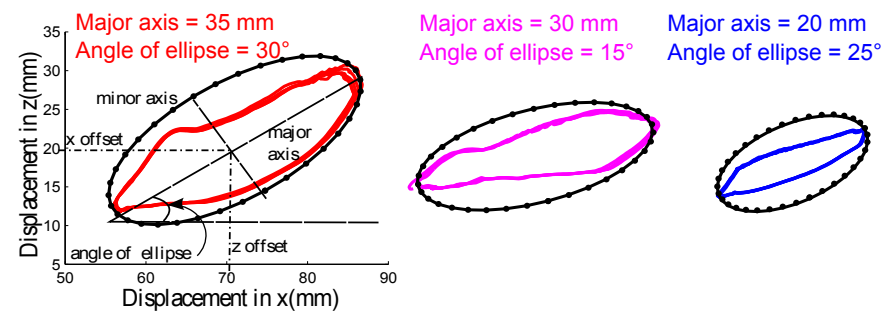

Fig. 5. Three ellipsoidal trajectories measured in vivo at the ankle. The shape of the trajectories is modulated via Labview by modifying the ellipse parameters: minor and major axis, angle of rotation, $\mathrm{x}$ and $\mathrm{z}$ offset values. of the actuators as well as the light-weight isostatic couplings, allow these deviations and motions outside of the sagittal plane to avoid harming the rodent. For example, we observe a rootmean-square deviation of $1.84 \mathrm{~mm}$ in the $\mathrm{x}$ direction in the top trajectory in Figure 5. The device allows guiding the limb of the rodent in airwalk configuration with a maximum guiding interaction force of $0.11 \pm 0.06 \mathrm{~N}$ measured with a six axis force/torque sensor placed at the ankle attachment. Also the results of this experiment show tracking errors of less than $20 \%$ with respect to the reference trajectory as shown in Figure 5. In recent applications of robot-enhanced therapy for rodents [28], the reported value in the best case for step length and height are less than $80 \%$ of the desired values during treadmill experiments. Hence, a $20 \%$ tracking error between the desired trajectory and the measured trajectory, as shown in Figure 5, is considered acceptable.

Preliminary results in this section prove that modulation of the trajectory of the subject's ankle with the exoskeleton is possible without an explicit model of the coupled dynamics of the interaction. One of the major benefits of soft actuation over other exoskeletons is that the compliance of the material accounts for the uncertainties and model discordances in the interaction which is achieved intuitively by human therapists but not by other mechanisms or control schemes.

\section{B. Ground Reaction Force (GRF) Modulation Tests with Foot Loading}

To register gait patterns, controlled force impulses to the rodent knee are given. The duration and amplitude of these impulses are unbridled since they are imparted solely by the personal experience of each researcher. Our device allows control of these aspects. We characterize the impact of shape parameters of the elliptic trajectories on the modulation of the vertical GRF when the animal is in contact with a force plate. For the foot loading experiments, the lower extremity of the exoskeleton was attached to the ankle of the rodent using a soft Fabrifoam $8 \mathrm{~mm}$ thick ankle sock instead of the cable tie. Although covered with neoprene, the cable tie used before triggered excessive co-contraction that imposed unwanted motions while impeding the required motion. A foambacked ankle attachment provides sufficient support without triggering adverse motions. Initially placed in contact with the force plate, ellipsoidal trajectories were imposed generating a pattern inspired by healthy rats' gait trajectories. The force and position in the 6D space were measured using the Vicon integrated system connected with the force plate. No EES or pharmacological agent was used during this experiment.

In the first few experiments we used a zip-tie as an ankle attachment. Although covered with neoprene, this triggered excessive co-contraction that imposed unwanted motions while impeding the required motion. In the later experiments, a foam-backed ankle attachment was used, which provided sufficient support without triggering adverse motions.

We achieve modulation of the GRF by changing the trajectory parameters such as the major axis, angle of rotation and the vertical offset in the operational space, as described in Figure 6 . The mean peak vertical ground reaction force can be 

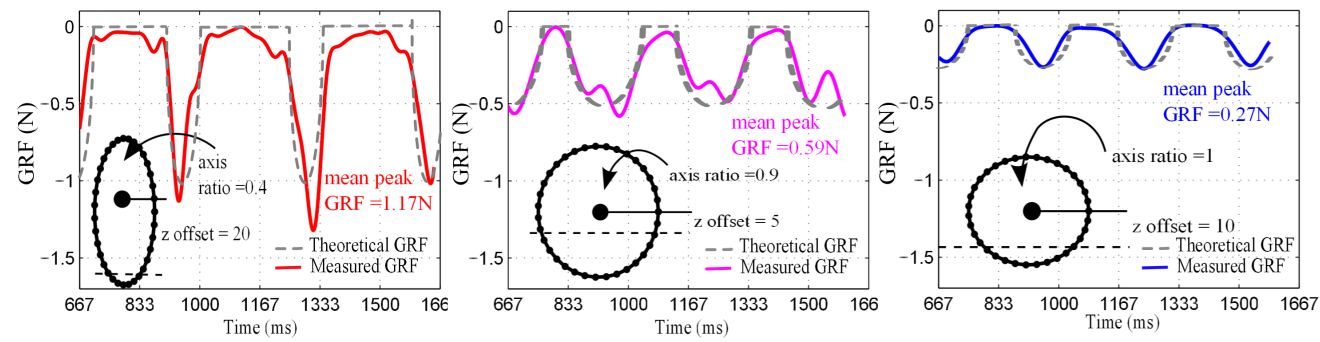

Fig. 6. Three vertical GRF profiles measured on a force plate. The modulation of the peak value was achieved by modifying the $\mathrm{z}$ offset value or the major axis value (here represented as the major vs minor axis ratio) of the input ellipse in the GUI. The dashed line represents the theoretical ground reaction force.

modulated up to near $1.2 \mathrm{~N}$. Also the profile of these forces can be modified by changing the form of the input ellipse. Namely, an ellipse that tends to be isotropic (with a ratio between major and minor axes close to 1) has a more sinusoidal force profile than the one generated by a "less round" ellipse as in Figure 6, where steep peaks are present. The profile of the forces applied reveal the nature of the interaction between the ankle guided by the exoskeleton and the ground. Therefore, the low impedance of the coupled system can be characterized and adapted by modifying actuator control and end-effector trajectories.

The effectiveness of the mechanical stimulation during locomotion training is evaluated by the loading that the therapist is able to impart to the rodent's leg during the stance phase such as to generate a swing. We have proven the capacity of our prototype to modulate the force transmitted to the ground through the rodent's extremity while being compliant and safe. Although two input parameters were analyzed, it is still necessary to study optimal trajectories to attain the desired force profile and gait pattern during training conditions.

\section{Ground Reaction Force (GRF) Modulation on a Treadmill}

Functional locomotor training after a spinal lesion is normally performed using a moving treadmill belt. In the previous sections, the stimulation was applied without this external disturbance. To identify the effect of the trajectory parameters when then rodent's leg is in contact with a moving treadmill, we showed that modulation of the gait's trajectory and ground reaction forces is possible even without explicit model of a disturbance. The introduction of a dragging force at the end effector of the exoskeleton generates a change in the overall response of the interaction. We should note that the rodent previously received locomotor training with manual assis-

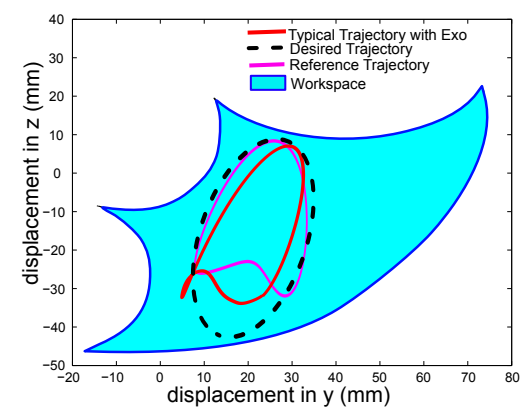

Fig. 7. The typical ankle trajectory within the exoskeleton workspace. The desired trajectory is an ellipsoidal approximation of the reference trajectory (in magenta) measured on a manually trained rodent after 5 weeks of training. tance while being attached to an automated, servo-controlled BWS system, and positioned over a motorized treadmill belt for bipedal locomotion. The animal was trained using the conventional scheme for spinalized subjects [1]. However, without these stimulations the rodent mainly drags its paws, but occasional spontaneous steps can occur. The retrained reflexes provoke the generation of these spontaneous steps.

The use of the exoskeleton dramatically changes the shape of these spontaneous steps by attracting the foot to predetermined elliptical paths which, as we observed in previous subsections, can be modulated in step length, step height and vertical ground reaction force. The exoskeleton-enabled modulation, in experiments in contact with the floor/treadmill, allows the therapist to adapt the training needs for different tasks such as climbing stairs, running, swimming etc. The introduction of pharmacological enablers without electrical stimulation can result in a lower reproducibility of limb kinematics [3]. Nevertheless, graded modulation of the step length, step height and ground reaction forces was achieved by choosing the parameters of the trajectory accordingly as in previous experiments in Subsections III-B and III-A. Figure 7 represents a typical trajectory measured on the ankle of the right paw of the rodent within the workspace of the exoskeleton. This curve is calculated as the mean trajectory over 10 consecutive steps. This is the longest streak of consecutive steps performed by the rodent before spastic movements appear. The spastic movements of the limbs are inevitable and the structure and actuation method used in the exoskeleton allows them, unlike other mechatronic solutions. These cycles were excluded from the computation of the mean trajectory. The standard deviation on the y-axis is $\pm 7.2 \mathrm{~mm}$ and $\pm 6.1 \mathrm{~mm}$ in the z-direction. Even if the treadmill acts as an output disturbance, the device succeeds in reshaping the trajectory of the ankle. As a means of comparison, a spontaneous step captured at the left paw that is not attached to the exoskeleton is depicted in Figure 7. Potentially coactivation of the leg muscles, which can lead to a rapid saturation of stepping movements caused by over stimulation can occur, but this phenomenon is observed rarely. Another challenge is that a discrepancy between the speed profiles of the "rodent step" and the imposed step may generate a resistance to motion in the form of a viscous field. In the case of a rigid exoskeleton this might potentially harm the rodent, unlike with the presented soft device.

In this experiment we demonstrated that, while using the exoskeleton, the rodent performs a cyclic "step-like" motion of the limb even without explicit modelling of the ground's 


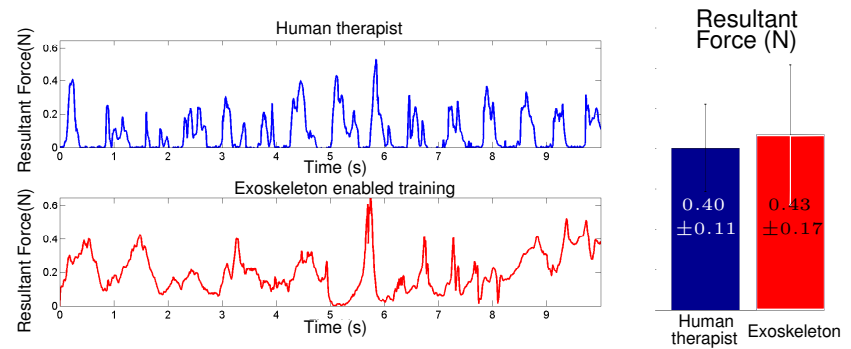

Fig. 8. Interaction resultant force comparison between manual training performed by a human therapist, exoskeleton guided airwalk test, and treadmill experiments with and without EES enabled stepping.

contact interaction properties. In order to get optimal behavior, there are a number of factors to take into account when analyzing the interaction dynamics of a rodent and a compliant mechanism with adaptable stiffness. For instance, a very low impedance at the interaction port may not trigger the gait motion of the limb. A high impedance on the contrary, can block the steps of the rodent. Also the effects of a viscous field in rehabilitation of lower extremities are not clear. The required impedance characterization of the system necessitates a more in-depth study with a larger number of subjects. Likewise, the visco-elastic properties of the material of SPA require more thorough modeling than that currently available [32]. Nevertheless, the observed actuation performance with disturbances shows a high potential for use in robots with physical interactions such as surgical tools that need to move with beating organs.

\section{Interaction forces comparison}

A preliminary test was performed to measure interaction forces at the interface between the exoskeleton and the ankle of the rodent as explained in subsection II-D. We compare the resultant force in three conditions of experiments to the force applied by the human therapist during traditional gait retraining.

In the previous subsection we showed that modulation of the stepping pattern can be achieved on the treadmill with the proposed exoskeleton. The interaction forces between the exoskeleton, that guides such stepping patterns, and the rodent are reported in Figure 8. The results show that the interaction forces of the active exoskeleton in treadmill-enabled training scenarios are comparable in order of magnitude to those applied manually by the therapist to the rodent's limb.

For an input trajectory of $30 \mathrm{~mm}$ in the major axis and an ellipse angle of $30^{\circ}$, the resultant force generated by the exoskeleton in the interaction port is $0.43 \pm 0.17 \mathrm{~N}$. The human therapist applies an average of $0.40 \pm 0.11 \mathrm{~N}$ for traditional training motion. Clearly, the trajectories of the paw are not the same but it should be noted that the therapist needs to be highly trained to be able to optimally adapt training to the requirements of each subject, and to comprehend the complex dynamics of reflex-based locomotor pattern generation.

\section{E. Gait period adaptation controller}

In treadmill experiments a rodent might modify the trajectory softly imposed by the exoskeleton. Our device allows back-drivability and increases the safety of the interaction but this softness has an impact on the effectiveness of the transmission of the forces to the ground, hence the loading of the foot. If a discrepancy in phase or period between the stimulation and the step of the system rodent+exoskeleton occurs, then a disturbance, in the form of a viscous field appears. Closing the loop to adapt the duration of the cycle is the first step towards implementing a full locomotor training controller that adapts input variables to kinematic and dynamic response of the rodent. By measuring the gait cycle we can minimize the disturbance perceived by the rodent. If the exoskeleton moves slower than the rodent, a visco-elastic field appears at the interaction port between the subject and the robot. On the contrary, if the exoskeleton is too fast with respect to the rodent, it might co-contract its muscles, impeding the desired gait. We developed and tested a gait period adaptation controller as described in Section II-E. Force and trajectory measured using the Vicon motion capture system and the 6axis force plate allows detection of the gait's frequency and triggers a frequency update function. It generates new patterns that tend to lower the difference in period between the two signals.

A preliminary study shows that reducing the difference between the gait's cycle and the input signal period, $\Delta P$, increases the level of force transmitted to the ground. In Figure 9a, a 3rd order curve fitting based on experimental data is applied to extrapolate the maximum Ground Reaction Force (GRF) if the offset $\Delta P$ is null. This corresponds to the required end-effector force summarized in Table I. The experimental data was obtained through a dozen tests with the same trajectory but changing the speed of the input ellipsoid. Each point in Figure 9a represents the mean force of at least 5 cycles. When the difference of the period is important, $>20 \%$ of the input period, the force transmission is less than $0.1 \mathrm{~N}, 10 \%$ of what is theoretically expected. On the contrary the GRF is near $0.6 \mathrm{~N}$ when the normalized $\Delta P$ is around 0.05. Although, GRF does not reach the level of the static footloading experiments detailed in Subsection III-B, the amelioration of the force transmission is a step forward towards increasing transparency. Figure $9 \mathrm{~b}$ shows a comparison between a fixed frequency response of the rodent+exoskeleton system in terms of kinematics and force transmitted to the ground, and an adapted period input signal response. The phase lag in the period between the two signals $(\Delta P)$ is reduced over the cycles. As a result the desired trajectory input signal frequency approximates the frequency of the measured ankle trajectory. Compared to the uncontrolled condition, the gait period adapted signal produces an increase in the mean peak vertical ground reaction force.

We focused on the control of the gait period pattern but not on the control of the SPA exoskeleton end effector position explicitly. This is because we provide assistance in a locomotion task that can be overtaken by the residual capacity of the subject rather than continuously correcting the trajectory imposing constraints onto the musculoskeletal system. As we don't separate the behavior of the rodent from the exoskeleton's, we do not require proprioceptive sensors such as encoders or integrated force sensors. This approach is based on an external 


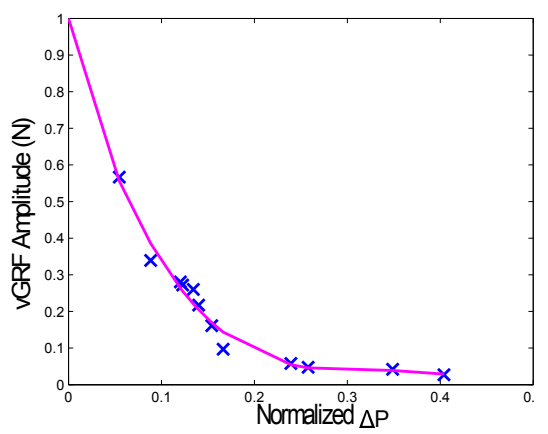

(a) Relationship between GRF mean peak amplitude vs normalized $\Delta P$
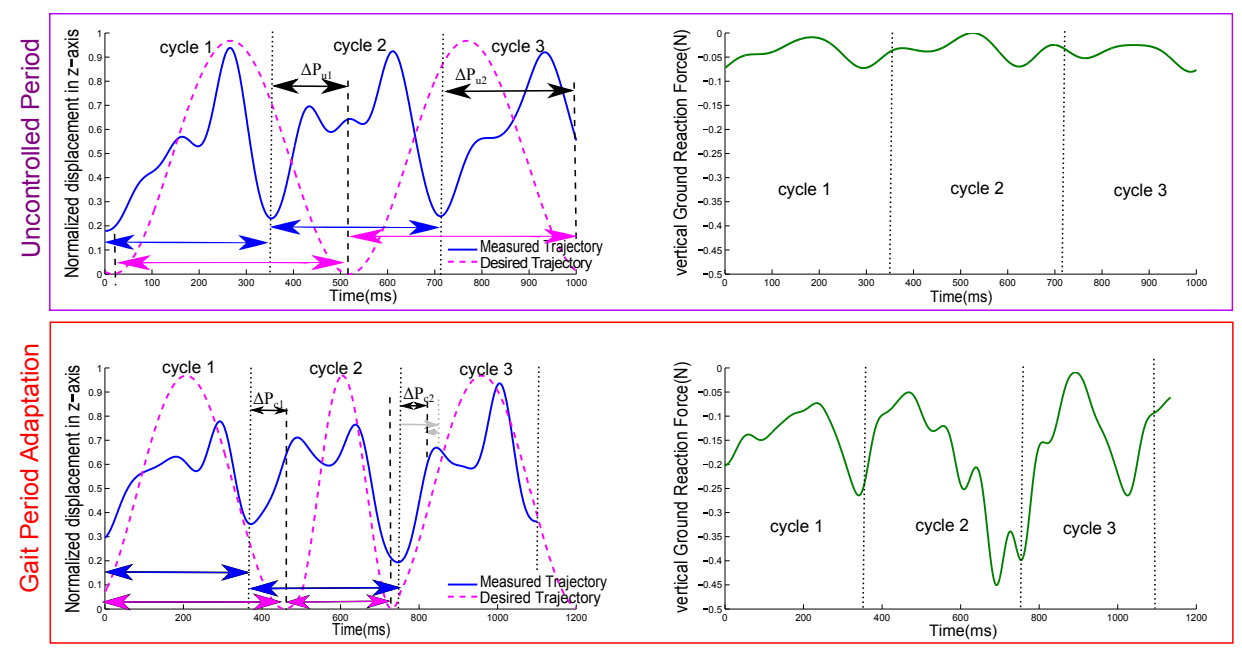

(b) Displacement and GRF with and without the gait period adaptation.

Fig. 9. Period Adaptation Controller results. (a) Best fit curve of the relation between GRF mean peak amplitude vs normalized $\Delta P$ (difference between the input signal period and the measured gait cycle period). Period adaptation results with and without the gait period adaptation (b). On the top, we see little change in the GRF due to the mismatch (large $\Delta P$ ) in the gait cycle between the exoskeleton and the subject. On the bottom, we see a dynamic array of GRFs for each cycle as the subject and the exoskeleton cycles match (small $\Delta P$ ).

sensor to modify the speed of actuation. Nevertheless, the system's structure allows integration of sensors if useful, as long as the inertia added to the moving parts that are in contact with fragile limbs is compensated for. Although the system is used in an openloop for the initial experiments in section III-A, the tracking error remains within an acceptable range of $20 \%$. During locomotion training on a treadmill, the modulation of the geometrical parameters of the trajectory is not sufficient to assure force transmission and hence foot loading. Even if the GRF level is successfully modulated between 0 and $0.6 \mathrm{~N}$ without adaptation of the actuator input pressure, exponential reduction of the capacity to transmit the forces applied to the leg towards the ground can be visualized in Figure 9a if the error between the input signal period and the actual gait period increases. This discrepancy between the periods affects the interaction and augments the impedance of the system regardless of the low structural stiffness of the actuation. Nevertheless, in perspective of implementing a fully automated functional locomotor training controller, this simple gait period (or frequency) adaptation controller constitutes the first step towards implementing impedance control and improving the quality of the interaction. Also by being reactive to changes in gait kinetics an "assistance-as-needed" can be implemented to provide suitable stimulation according to therapist's orders and respecting the evolution of the subject.

\section{Discussion AND CONCLUSIONS}

In this paper, we present a robotic exoskeleton for rodents exploiting the SPAs' inherent compliance and performance to provide mechanical stimulation during BWS treadmill training to rodent's who have undergone SCI. The device presented in this paper exerts controlled mechanical stimulation that allows paralyzed rats to produce a broad range of foot trajectories during continuous locomotion whilst showing that compliance due to the low stiffness of the actuators and fixations affords adaptation to the constraints imposed by the subject. The preliminary tests reported in this paper demonstrate the capacity of the hardware and the implemented control strategy to satisfy the functional requirements of a rehabilitative wearable device for rodents. Specifically, the proposed device is able to apply a $1 \mathrm{~N}$ force to the end effector (the ankle) at 2 cycles/s throughout the defined workspace. We show trajectory imposition and modulation of the path of the limb through actuation of the exoskeleton, for example, in airwalk experiments when the rodent paw is not in contact with the ground, an interaction force of $0.11 \pm 0.06 \mathrm{~N}$ is sufficient to drag the ankle to an ellipsoidal trajectory with an error rate of less than $20 \%$. In treadmill experiments, where the EES triggered the swing phase of the limb, the measured interaction force is $0.43 \pm 0.17 \mathrm{~N}$. This shows the capacity of the system for loading of the foot and modulation of such forces using SPAs as a mode of actuation. The exoskeleton also successfully improves the transmission of mechanical inputs to the limb by adapting the period of the gait cycle to compensate for a lag caused by the residual capacity of the rodent when no EES is applied, and is able to trigger the swing phase. Moreover, it maintains a tracking error with respect to the desired trajectory inferior to $30 \%$, while increasing the GRF around four times compared to without the adaptation controller. Adaptation of the system to extrinsic constraints [17] generated by the rodent while satisfying the functional requirements of a task is a major advantage of soft robotic systems over conventional rigid non-backdrivable actuated mechanisms.

Regarding the speed of stimulation, the frequencydependent modulation was consistent across experiments, 
showing that the prototype soft exoskeleton provides an output bandwidth of up to $2 \mathrm{~Hz}$ on average. We should note that the classical manual training method has low bandwidth since the therapist can only apply controlled forces up to $1 \mathrm{~Hz}$ following a desired trajectory [38]. The prototype however can apply controlled forces of $1 \mathrm{~N}$ per foot at twice the frequency of a human therapist. Furthermore, by adapting the period of the input signal that generates the exoskeleton's motion, we were able to modulate the ground reaction forces and hence improve the quality of the interaction. Indeed, precisely identifying the cycle phases and controlling the stimulation period enables the introduction of the "assistance-as-needed" concept. It also constitutes the first step towards implementation of a dynamic impedance control strategy necessary to enable a fully automated assistance system, such as the foreseen robot controller, illustrated in Figure 10. The system could adapt the impedance of the actuators using the inverse dynamics model of the exoskeleton, while a forward model of the sensorimotor coupling allows adjusting the EES patterns in real time [29]. By monitoring the kinematics, GRF, and EMG signals, we will be able to exert AAN force loading to directly evaluate the subject's effort and increase transparency of the whole system.

In effect, the overall system aims at improving the physical interaction between the rodent and the robot (ergo the quality of functional training) by taking advantage of the synergies between different modes of stimulation. A broad clinical evaluation will be needed in order to implement more complex assistance strategies mixing EES neuro-modulation with automated exoskeleton-enabled retraining and to prove the therapeutic effects across days and across rats of this novel AAN controller concept. As a continuation of the presented work, the trajectories should be adapted to previously defined suitable therapeutic gait patterns and adjusted to appropriately support only in case of weakness in terms of load sharing. Further research should also include controllable stiffness for the SPAs in order to achieve impedance control of the structure. Next, we will be implementing a multi-stimulation controller involving synergies between EES and mechanical stimulation to improve the quality of functional training.

In addition to its application as a soft exoskeleton for rodents, the SPA-based robots signify advancement for general human-robot interactive devices [27], [24]. This biomimetic approach (rigid links connected to passive joints actuated by flexible and soft SPAs), allows a large diversity in interaction with unstructured environments and facilitates scaling design of SPAs to match human size criteria. Deformable materials enable flexible motion of the kinematic chain and selforganization to match the body motion of the human in contact with it. In many cases, physical processes are much faster than any controlled mechanism with limited DoF. The complex interaction between two decision making agents (human and robot) can be computationally expensive to model and often limited by the hardware. The idea that certain aspects of the computation can be compensated by the morphological and material components could in fact simplify computation while also increasing the reaction speed [46]. The presented soft exoskeleton illustrates a highly customizable proof-of-concept of the use of soft pneumatic actuators in direct interaction with rodents that can serve as a versatile platform for a more complex functional training.

\section{ACKNOWLEDGEMENTS}

This work is funded by the Swiss National Centre of Competence in Research (NCCR) in Robotics.

\section{REFERENCES}

[1] G. Courtine, Y. Gerasimenko, R. van den Brand, A. Yew, P. Musienko, H. Zhong, B. Song, Y. Ao, R. M. Ichiyama, I. Lavrov et al., "Transformation of nonfunctional spinal circuits into functional states after the loss of brain input," Nature neuroscience, vol. 12, no. 10, pp. 13331342, 2009.

[2] J. Cha, C. Heng, D. J. Reinkensmeyer, R. R. Roy, V. R. Edgerton, and R. D. De Leon, "Locomotor ability in spinal rats is dependent on the amount of activity imposed on the hindlimbs during treadmill training," Journal of neurotrauma, vol. 24, no. 6, pp. 1000-1012, 2007.

[3] R. Van den Brand, J. Heutschi, Q. Barraud, J. DiGiovanna, K. Bartholdi, M. Huerlimann, L. Friedli, I. Vollenweider, E. M. Moraud, S. Duis, N. Dominici, S. Micera, P. Musienko, and G. Courtine, "Restoring voluntary control of locomotion after paralyzing spinal cord injury," Science, vol. 336, no. 6085, pp. 1182-1185, 2012.

[4] S. Jezernik, G. Colombo, T. Keller, H. Frueh, and M. Morari, "Robotic orthosis lokomat: A rehabilitation and research tool," Neuromodulation: Technology at the neural interface, vol. 6, no. 2, pp. 108-115, 2003.

[5] S. K. Banala, S. H. Kim, S. K. Agrawal, and J. P. Scholz, "Robot assisted gait training with active leg exoskeleton (alex)," Neural Systems and Rehabilitation Engineering, IEEE Transactions on, vol. 17, no. 1, pp. 2-8, 2009.

[6] D. J. Reinkensmeyer, D. Aoyagi, J. L. Emken, J. A. Galvez, W. Ichinose, G. Kerdanyan, S. Maneekobkunwong, K. Minakata, J. A. Nessler, R. Weber et al., "Tools for understanding and optimizing robotic gait training," Journal of rehabilitation research and development, vol. 43, no. 5, p. 657, 2006

[7] A. Schiele and F. C. van der Helm, "Kinematic design to improve ergonomics in human machine interaction," Neural Systems and Rehabilitation Engineering, IEEE Transactions on, vol. 14, no. 4, pp. 456-469, 2006.

[8] H. I. Krebs, J. J. Palazzolo, L. Dipietro, M. Ferraro, J. Krol, K. Rannekleiv, B. T. Volpe, and N. Hogan, "Rehabilitation robotics: Performancebased progressive robot-assisted therapy," Autonomous Robots, vol. 15, no. 1, pp. 7-20, 2003.

[9] R. Riener, L. Lünenburger, S. Jezernik, M. Anderschitz, G. Colombo, and V. Dietz, "Patient-cooperative strategies for robot-aided treadmill training: first experimental results," Neural Systems and Rehabilitation Engineering, IEEE Transactions on, vol. 13, no. 3, pp. 380-394, 2005.

[10] E. T. Wolbrecht, V. Chan, V. Le, S. C. Cramer, D. J. Reinkensmeyer, and J. E. Bobrow, "Real-time computer modeling of weakness following stroke optimizes robotic assistance for movement therapy," in Neural Engineering, 2007. CNE'07. 3rd International IEEE/EMBS Conference on. IEEE, 2007, pp. 152-158.

[11] J. A. Nessler, W. Timoszyk, M. Merlo, J. L. Emken, K. Minakata, R. R. Roy, R. D. de Leon, V. R. Edgerton, and D. J. Reinkensmeyer, "A robotic device for studying rodent locomotion after spinal cord injury," IEEE Transactions on neural systems and rehabilitation engineering, vol. 13, no. 4, 2005.

[12] D. J. Reinkensmeyer, W. K. Timoszyk, R. D. de Leon, R. Joynes, E. Kwak, K. Minakata, and V. R. Edgerton, "A robotic stepper for retraining locomotion in spinal-injured rodents," in Robotics and Automation, 2000. Proceedings. ICRA'OO. IEEE International Conference on, vol. 3. IEEE, 2000, pp. 2889-2894.

[13] Y. S. Song, "Design, implementation and validation of an exoskeletal robot for locomotion studies in rodents," $\mathrm{Ph}$.D. dissertation, Massachusetts Institute of Technology, June 2012.

[14] E. T. Roche, R. Wohlfarth, J. T. B. Overvelde, N. V. Vasilyev, F. A. Pigula, D. J. Mooney, K. Bertoldi, and C. J. Walsh, "A bioinspired soft actuated material," Advanced Materials, vol. 26, no. 8, pp. 1145-1145, 2014.

[15] B. Mosadegh, P. Polygerinos, C. Keplinger, S. Wennstedt, R. F. Shepherd, U. Gupta, J. Shim, K. Bertoldi, C. J. Walsh, and G. M. Whitesides, "Pneumatic networks for soft robotics that actuate rapidly," Advanced Functional Materials, vol. 24, no. 15, pp. 2163-2170, 2014. 


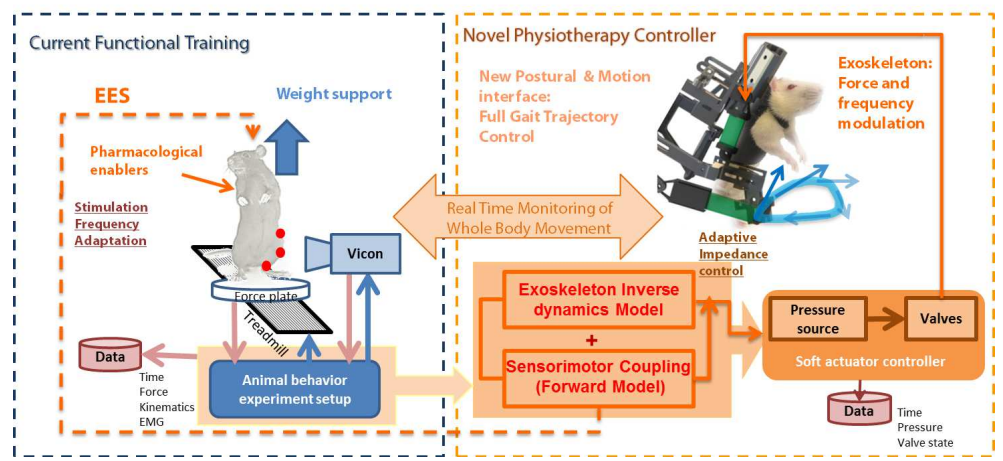

Fig. 10. Prospective full in vivo physiotherapy control scheme. Real time kinematics and force values measured during functional training are used to modulate frequency of EES pulses capitalizing a forward model of sensorimotor coupling. This information is also used to adapt the impedance of the electro-pneumatically controlled soft exoskeleton.

[16] R. Deimel and O. Brock, "A compliant hand based on a novel pneumatic actuator," in Robotics and Automation (ICRA), 2013 IEEE International Conference on. IEEE, 2013, pp. 2047-2053.

[17] C. Eppner, R. Deimel, J. Alvarez-Ruiz, M. Maertens, and O. Brock, "Exploitation of environmental constraints in human and robotic grasping," The International Journal of Robotics Research, vol. 34, no. 7, pp. 1021-1038, June 2015.

[18] M. T. Tolley, R. F. Shepherd, B. Mosadegh, K. C. Galloway, M. Wehner, M. Karpelson, R. J. Wood, and G. M. Whitesides, "A resilient, untethered soft robot," Soft Robotics, vol. 1, no. 3, pp. 213-223, 2014.

[19] J. M. Florez, B. Shih, Y. Bai, and J. Paik, "Soft pneumatic actuators for legged locomotion," in International Conference on Robotics and Biomimetics, December 2014, p. to appear.

[20] D. P. Holland, E. J. Park, P. Polygerinos, G. J. Bennett, and C. J. Walsh, "The soft robotics toolkit: Shared resources for research and design," Soft Robotics, vol. 1, no. 3, pp. 224-230, 2014.

[21] Y. S. Song, Y. Sun, R. van den Brand, J. von Zitzewitz, S. Micera, G. Courtine, and J. Paik, "Soft robot for gait rehabilitation of spinalized rodents," in Intelligent Robots and Systems (IROS), 2013 IEEE/RSJ International Conference on, Nov 2013, pp. 971-976.

[22] P. Maeder-York, T. Clites, E. Boggs, R. Neff, P. Polygerinos, D. Holland, L. Stirling, K. Galloway, C. Wee, and C. Walsh, "Biologically inspired soft robot for thumb rehabilitation," Journal of Medical Devices, vol. 8, no. 2, p. 020933, 2014.

[23] Y.-L. Park, B.-r. Chen, D. Young, L. Stirling, R. J. Wood, E. Goldfield, and R. Nagpal, "Bio-inspired active soft orthotic device for ankle foot pathologies," in Intelligent Robots and Systems (IROS), 2011 IEEE/RSJ International Conference on. IEEE, 2011, pp. 4488-4495.

[24] M. Wehner, B. Quinlivan, P. M. Aubin, E. Martinez-Villalpando, M. Baumann, L. Stirling, K. Holt, R. Wood, and C. Walsh, "A lightweight soft exosuit for gait assistance," in Robotics and Automation (ICRA), 2013 IEEE International Conference on. IEEE, 2013, pp. 3362-3369.

[25] D. Sasaki, T. Noritsugu, and M. Takaiwa, "Development of active support splint driven by pneumatic soft actuator (assist)," in Robotics and Automation, 2005. ICRA 2005. Proceedings of the 2005 IEEE International Conference on. IEEE, 2005, pp. 520-525.

[26] A. Asbeck, R. Dyer, A. Larusson, and C. Walsh, "Biologically-inspired soft exosuit," in Rehabilitation Robotics (ICORR), 2013 IEEE International Conference on, June 2013, pp. 1-8.

[27] P. Polygerinos, S. Lyne, Z. Wang, L. F. Nicolini, B. Mosadegh, G. M. Whitesides, and C. J. Walsh, "Towards a soft pneumatic glove for hand rehabilitation," in Intelligent Robots and Systems (IROS), 2013 IEEE/RSJ International Conference on. IEEE, 2013, pp. 1512-1517.

[28] C. Lee, D. Won, M. J. Cantoria, M. Hamlin, and R. D. de Leon, "Robotic assistance that encourages the generation of stepping rather than fully assisting movements is best for learning to step in spinally contused rats," Journal of neurophysiology, vol. 105, no. 6, pp. 2764-2771, 2011.

[29] N. Wenger, E. Martin Moraud, S. Raspopovic, M. Bonizzato, J. DiGiovanna, P. Musienko, M. Morari, S. Micera, and G. Courtine, "Closedloop neuromodulation of spinal sensorimotor circuits controls refined locomotion after complete spinal cord injury," Science Translational Medicine, vol. 6, no. 255, 2014.

[30] W. L. Johnson, D. L. Jindrich, R. R. Roy, and V. Reggie Edgerton, "A three-dimensional model of the rat hindlimb: musculoskeletal geometry and muscle moment arms," Journal of biomechanics, vol. 41, no. 3, pp. 610-619, 2008.
[31] F. Connolly, P. Polygerinos, C. J. Walsh, and K. Bertoldi, "Mechanical programming of soft actuators by varying fiber angle," Soft Robotics, vol. 2 , no. 1, pp. 26-32, 2015.

[32] Y. Elsayed, A. Vincensi, C. Lekakou, T. Geng, C. Saaj, T. Ranzani, M. Cianchetti, and A. Menciassi, "Finite element analysis and design optimization of a pneumatically actuating silicone module for robotic surgery applications," Soft Robotics, vol. 1, no. 4, pp. 255-262, 2014.

[33] P. Polygerinos, Z. Wang, J. Overvelde, K. Galloway, R. Wood, K. Bertoldi, and C. Walsh, "Modeling of soft fiber-reinforced bending actuators," Robotics, IEEE Transactions on, vol. 31, no. 3, pp. 778-789, June 2015.

[34] J. P. Sun Yi, Yun Seong Song, "Characterization of silicone rubber based soft pneumatic actuators," in International Conference on Intelligent Robots and Systems, November 2013, pp. 4446-4453.

[35] M. Cianchetti, A. Arienti, M. Follador, B. Mazzolai, P. Dario, and C. Laschi, "Design concept and validation of a robotic arm inspired by the octopus," Materials Science and Engineering: $C$, vol. 31, no. 6, pp. 1230-1239, 2011.

[36] R. F. Shepherd, F. Ilievski, W. Choi, S. A. Morin, A. A. Stokes, A. D. Mazzeo, X. Chen, M. Wang, and G. M. Whitesides, "Multigait soft robot," Proceedings of the National Academy of Sciences, vol. 108, no. 51, pp. 20 400-20 403, 2011.

[37] R. F. Shepherd, A. A. Stokes, R. Nunes, and G. M. Whitesides, "Soft machines that are resistant to puncture and that self seal," Advanced Materials, vol. 25, no. 46, pp. 6709-6713, 2013.

[38] V. Falk, "Manual control and trackinga human factor analysis relevant for beating heart surgery," The Annals of thoracic surgery, vol. 74, no. 2, pp. 624-628, 2002.

[39] T. Lenzi, S. M. M. D. Rossi, N. Vitiello, and M. C. Carrozza, "Intentionbased emg control for powered exoskeletons," Biomedical Engineering, IEEE Transactions on, vol. 59, no. 8, pp. 2180-2190, 2012.

[40] T. S. Buchanan, D. G. Lloyd, K. Manal, and T. F. Besier, "Neuromusculoskeletal modeling: estimation of muscle forces and joint moments and movements from measurements of neural command," Journal of applied biomechanics, vol. 20, no. 4, p. 367, 2004.

[41] J. R. Wolpaw, N. Birbaumer, D. J. McFarland, G. Pfurtscheller, and T. M. Vaughan, "Brain-computer interfaces for communication and control," Clinical neurophysiology, vol. 113, no. 6, pp. 767-791, 2002.

[42] S. M. M. De Rossi, N. Vitiello, T. Lenzi, R. Ronsse, B. Koopman, A. Persichetti, F. Vecchi, A. J. Ijspeert, H. Van der Kooij, and M. C. Carrozza, "Sensing pressure distribution on a lower-limb exoskeleton physical human-machine interface," Sensors, vol. 11, no. 1, pp. 207227, 2010.

[43] T. Lenzi, N. Vitiello, S. M. M. De Rossi, A. Persichetti, F. Giovacchini, S. Roccella, F. Vecchi, and M. C. Carrozza, "Measuring human-robot interaction on wearable robots: A distributed approach," Mechatronics, vol. 21, no. 6, pp. 1123-1131, 2011.

[44] L. Marchal-Crespo and D. J. Reinkensmeyer, "Review of control strategies for robotic movement training after neurologic injury," Journal of neuroengineering and rehabilitation, vol. 6, no. 1, p. 20, 2009.

[45] R. Ronsse, N. Vitiello, T. Lenzi, J. van den Kieboom, M. C. Carrozza, and A. J. Ijspeert, "Human-robot synchrony: flexible assistance using adaptive oscillators," Biomedical Engineering, IEEE Transactions on, vol. 58, no. 4, pp. 1001-1012, 2011.

[46] R. Pfeifer, M. Lungarella, and F. Iida, "The challenges ahead for bioinspired'soft'robotics," Communications of the ACM, vol. 55, no. 11, pp. 76-87, 2012. 